\title{
LOCALIZAÇÃO DOS SULCOS E GIROS DA FACE SÚPERO-LATERAL DO CÉREBRO NA TOMOGRAFIA COMPUTADORIZADA E NA RESSONÂNCIA MAGNÉTICA
}

\author{
Sebastião Gusmão', Guilherme Carvalhal Ribas², Roberto Leal Silveira³, Uedsom Tazinaffo ${ }^{4}$
}

\begin{abstract}
RESUMO - O conhecimento da anatomia da face súpero-lateral do cérebro é fundamental para localizar e abordar as lesões cerebrais. Faz-se a revisão da literatura dos sinais e referências para identificar os sulcos e giros da face súpero-lateral do cérebro na tomografia computadorizada e na ressonância magnética. 0 uso conjunto dos vários sinais possibilita a identificação dos sulcos e giros e a localização das lesões cerebrais na quase totalidade dos casos.
\end{abstract}

PALAVRAS-CHAVE: anatomia cerebral, sulcos cerebrais, sulco central, tomografia computadorizada, ressonância magnética.

The sulci and gyri localization of the brain superolateral surface in computed tomography and magnetic resonance imaging

\begin{abstract}
The knowledge of the superolateral surface brain anatomy is fundamental to localize and to approach the cerebral lesions. A literature review of the signs and landmarks for the identification of its sulci and gyri in computed tomography and magnetic resonance imaging had been done. The combined use of these several signs makes possible the sulci and gyri identification and its cerebral lesions localization in almost all cases.
\end{abstract}

KEY WORDS: brain anatomy, cerebral sulci, central sulcus, computed tomography, magnetic resonance imaging.

Apesar de a superfície dos hemisférios cerebrais apresentar grande variabilidade, os principais sulcos, formados na vida fetal, têm localizações relativamente constantes, propiciando referências precisas ${ }^{1}$. Como o córtex cerebral tem localizações funcionais altamente específicas, a identificação dos sulcos e giros é de grande importância clínica e cirúrgica. Essa identificação possibilita a compreensão da anatomia da superfície súpero-lateral (convexidade) do cérebro, a localização de lesões, o planejamento cirúrgico e as vias para a abordagem transsulcal.

Os sulcos e giros da porção superior (medial ou parasagital) da convexidade cerebral são melhor identificados nos cortes axiais da tomografia computadorizada (TC) e da ressonância magnética (RM), e os da porção inferior (lateral ou perisilviana), nos cortes sagitais laterais da RM.

O objetivo do presente estudo é fazer a revisão da literatura dos sinais e referências para identificar os sulcos e giros da convexidade cerebral na TC e na RM.

A revisão da literatura foi realizada por meio da Medline, cruzando-se os termos brain anatomy, cerebral sulci, central sulcus, computed tomography, magnetic resonance imaging no período de 1971 (data da introdução da TC) a 1999.

\section{BASES ANATÔMICAS}

A convexidade cerebral é delimitada acima pela borda súpero-medial do hemisfério cerebral, abaixo pela borda ínfero-lateral e, à frente, pela borda supraciliar.

A anatomia da convexidade cerebral é melhor sistematizada se se adota o conceito de lobo central interposto entre os lobos frontal e parietal. O lobo central compreende os giros pré ( $\mathrm{GPrC}$ ) e pós-central (GPoC), dispostos obliquamente e delimitados pelos

\footnotetext{
'Professor Adjunto do Departamento de Psiquiatria e Neurologia da Faculdade de Medicina da Universidade Federal de Minas Gerais; ${ }^{2}$ Coordenador do Setor de Neuroanatomia Aplicada da Disciplina de Topografia Estrutural Humana do Departamento de Cirurgia da Faculdade de Medicina da Universidade de São Paulo; ${ }^{3}$ Coordenador do Serviço de Neurocirurgia do Hospital Madre Teresa; ${ }^{4}$ Coordenador do Serviço de Imagiologia do Hospital Madre Teresa
}

Recebido 1 Agosto 2000, recebido na forma final 22 Setembro 2000. Aceito 27 Setembro 2000. 


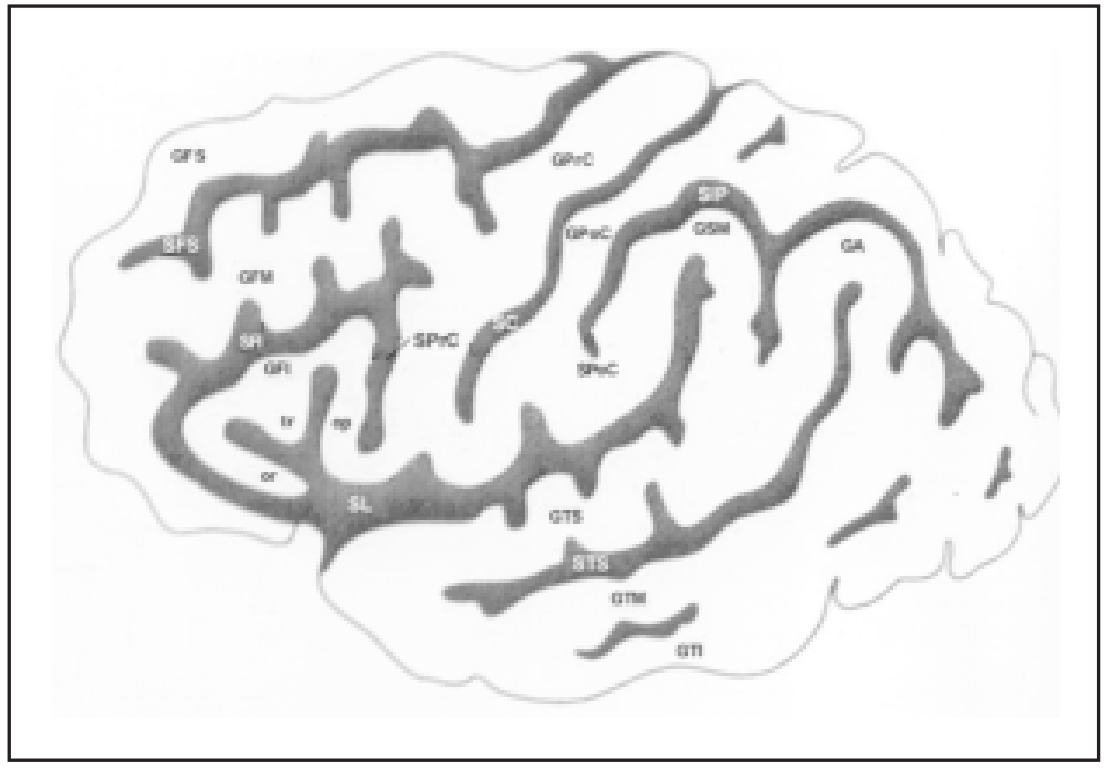

Fig 1. Esquema dos sulcos e giros da face súpero-lateral do cérebro. SL: sulco lateral; SFI: sulco frontal inferior; SFS: sulco frontal superior; SPrC: sulco pré-central; SC: sulco central; SpoC: sulco pós-central; SIP: sulco intraparietal; STS: sulco temporal superior; GFI: giro frontal inferior; Or: parte orbitária do GFI; T: parte triangular do GFI; Op: parte opercular do GFI; GFM: giro frontal médio; GFS: giro frontal superior; GPrC: giro précentral; GPoC: giro pós-central; GSM: giro supramarginal; GA: giro angular; GTS: giro temporal superior; GTM: giro temporal médio; GTI: giro temporal inferior. sulcos pré-central (SPrC), central (SC) e pós-central (SpoC). Esses giros dobram-se sobre a borda súperomedial do hemisfério cerebral e fundem-se em torno da extremidade superior e medial do SC para formar o lóbulo paracentral. Esse lóbulo é delimitado, posteriormente, pela pars marginalis (PM) do sulco do giro do cíngulo, situada logo à frente da extremidade superior do SPoC. Inferiormente, próximo à fissura silviana ou sulco lateral (SL), o GPrC e o GPoC fundem-se para formar o giro subcentral (Figs 1, 2 e 3).

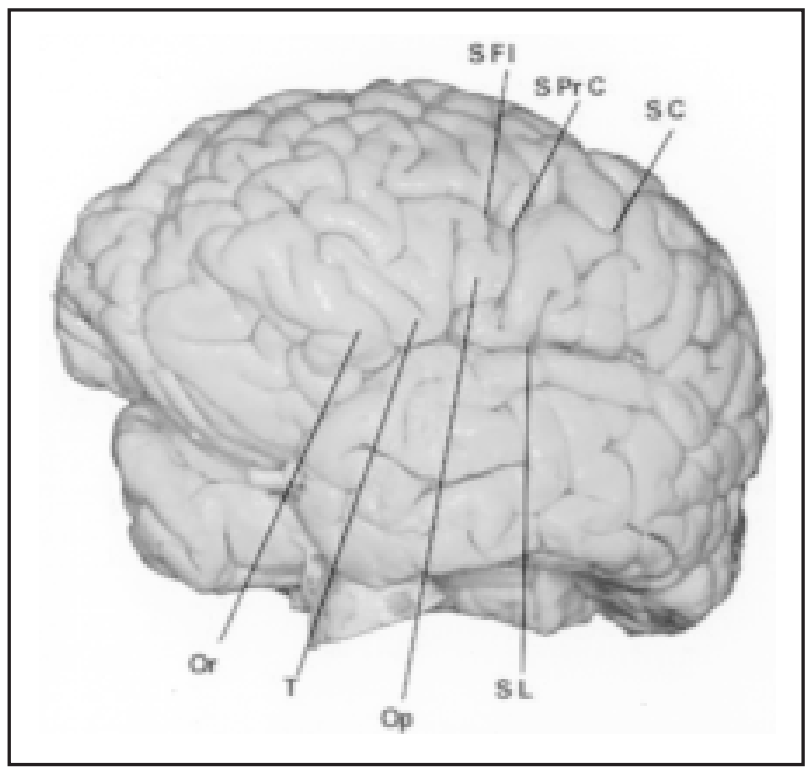

Fig 2. Vista da superfície súpero-lateral do cérebro. SL: sulco lateral; Or: parte orbitária do giro frontal inferior; $T$ : parte triangular do giro frontal inferior; Op: parte opercular do giro frontal inferior; SC: sulco central; SFI: sulco frontal inferior; SPrC: sulco pré-central.
Acima do SL e anterior ao SPrC, o lobo frontal é formado pelos giros frontais superior (GFS), médio (GFM) e inferior (GFI), dispostos horizontalmente e separados pelos sulcos frontais superior (SFS) e inferior (SFI). Posterior ao SPoC, e anterior à linha imaginária que une o sulco parieto-occipital à incisura pré-occipital, encontra-se o lobo parietal, que é subdividido pelo sulco intraparietal (SIP) nos lóbulos parietais superior e inferior. O lóbulo parietal inferior é subdividido nos giros supramarginal (GSM), ante-

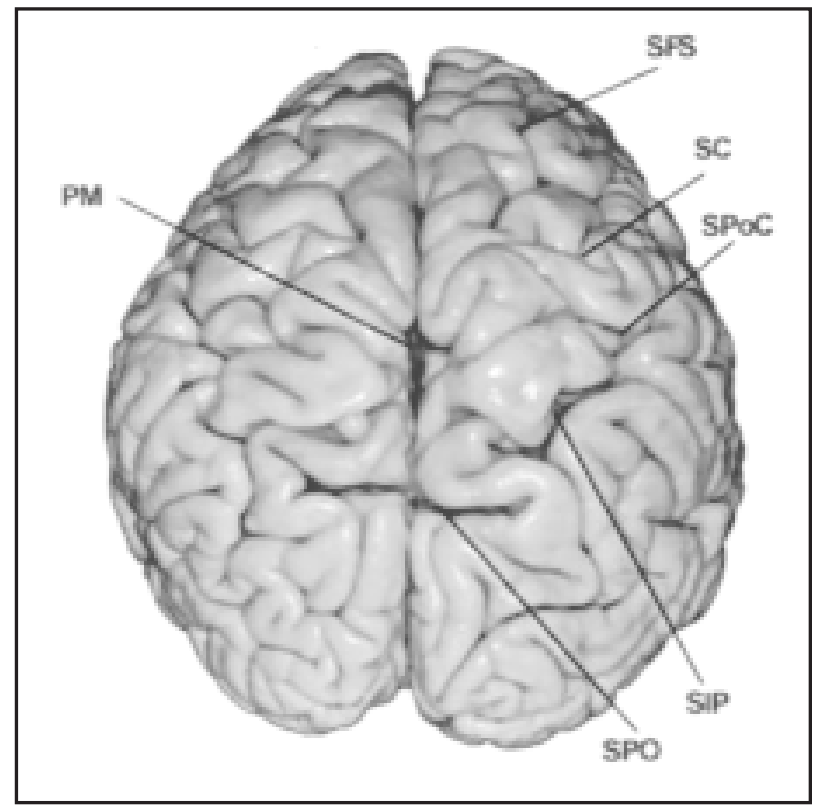

Fig 3. Vista súpero-posterior das superfícies laterais dos hemisférios cerebrais. PM: pars marginalis do sulco do giro do cíngulo; SFS: sulco frontal superior; SC: sulco central; SPoC: sulco pós-central; SIP: sulco intraparietal; SPO: sulco parieto-occipital. 
riormente, e angular (GA), posteriormente. Posterior à linha de divisão dos lobos parietal e occipital, encontra-se a face lateral do lobo occipital, formada por três pequenos giros (superior, médio e inferior), dispostos longitudinalmente (Figs 1,2 e 3).

Abaixo do SL a face súpero-lateral do lobo temporal é formada pelos giros temporais superior (GTS), médio (GTM) e inferior (GTI), delimitados pelos sulcos temporais superior (STS) e inferior (STI). O GTS funde-se com o lóbulo parietal inferior, e o GTM e o GTI, com o lobo occipital. Os lobos temporal e occipital são delimitados pela metade inferior da linha imaginária que liga a extremidade superior do sulco parieto-occipital à incisura pré-occipital (Figs 1 e 2).

\section{CONVEXIDADE CEREBRAL ALTA (MEDIAL OU PARASAGITAL)}

Os seguintes sinais para localização dos sulcos e giros da convexidade cerebral alta nos cortes axiais da TC e da RM foram descritos.

\section{1 - Término do SFS no SPrC}

O SFS cursa paralelamente e, aproximadamente, a $2,1 \mathrm{~cm}$ da fissura inter-hemisférica, geralmente como uma linha interrompida, e termina em ângulo reto no $\mathrm{SPrC}^{2}$. O SFS é bem visibilizado em $85 \%$ na TC e em $100 \%$ na RM. Ele termina no SPrC em $82 \%$ na TC e em $88 \%$ na RM (Figs 1, 3 e 4$)^{3}$.

\section{2 - Relação do SPrC e SC com a sutura coronária}

A sutura coronária é aproximadamente perpendicular à linha médio-sagital (sutura sagital) no trajeto do bregma (união das suturas coronária e sagital) ao estefânio (ponto de união da sutura coronária com a linha temporal superior). A partir desse ponto, ela curva-se progressivamente para frente até alcançar o ptério. Na TC, a sutura coronária aparece como entalhe na tábua interna, determinado pela transição dos ossos frontal e parietal, e apresenta maior atenuação (densidade) em relação ao restante do osso.

O SPrC apresenta curso descendente anterior na convexidade frontal, dispondo-se da borda súperomedial do hemisfério ao SL. Em sua extremidade superior, encontra-se a $2,8 \mathrm{~cm}$ da sutura coronária (bregma $)^{4}$. Em cortes tomográficos, essa distância é de $2,6 \mathrm{~cm}^{2}$. Em sua porção média, o SPrC aproxima-se da sutura coronária, cursando logo posterior a ela até o $\mathrm{SL}^{5}$.

O SPrC aparece nos cortes axiais altos como linha oblíqua dirigida no sentido póstero-anterior e da linha média para o entalhe da sutura coronária, a aproximadamente $2,6 \mathrm{~cm}$ posterior a esta sutura ${ }^{2}$ (Fig 4).

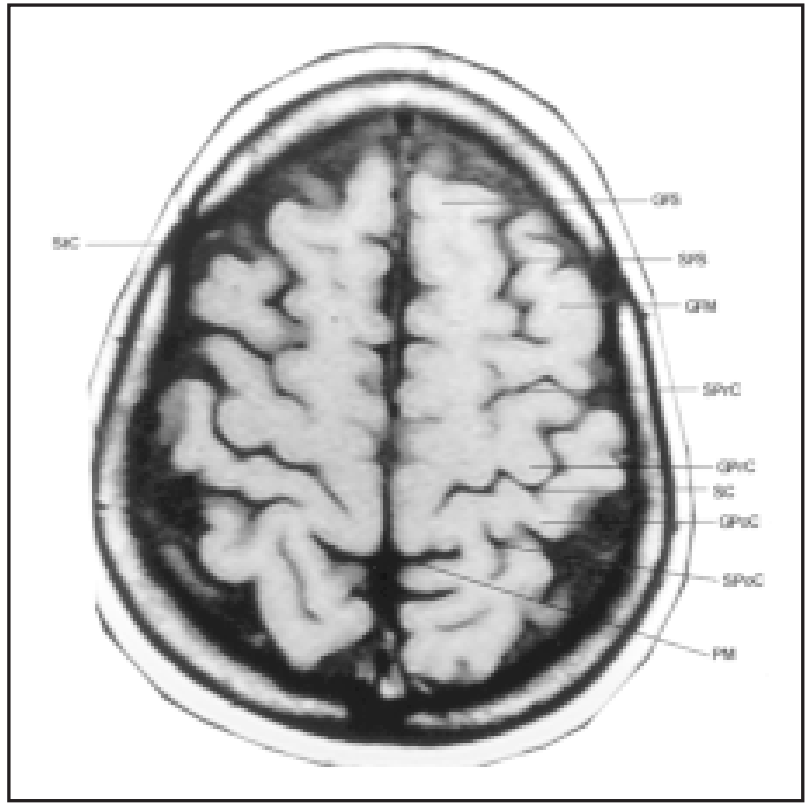

Fig 4. Corte axial da ressonância magnética mostrando a convexidade cerebral alta (parasagital). SFS: sulco frontal superior; SPrC: sulco pré-central; SC: sulco central; SpoC: sulco pós-central; PM: pars marginalis do sulco do giro do cíngulo; GFS: giro frontal superior; GFM: giro frontal médio; GPrC: giro pré-central; GPoC: giro pós-central; StC: sutura coronária.

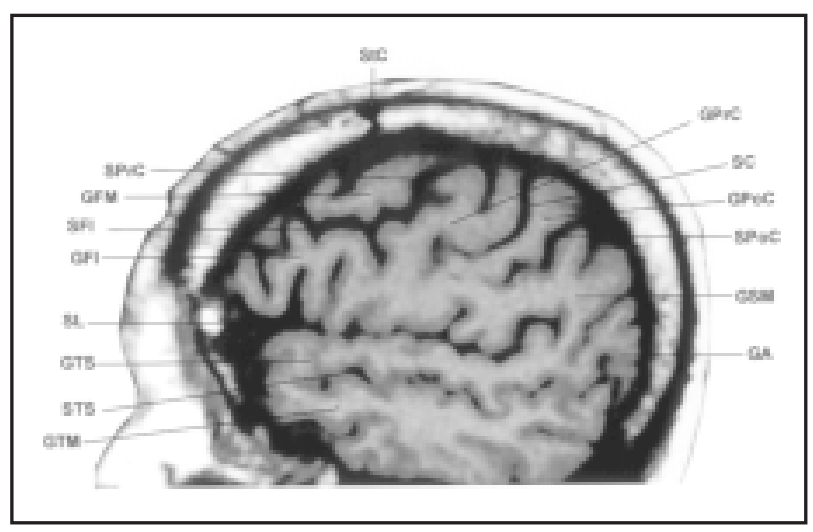

Fig 5. Corte sagital da ressonância magnética mostrando a convexidade cerebral baixa (perisilviana). SL: sulco lateral; SFI: sulco frontal inferior; SPrC: sulco pré-central; SC: sulco central; SpoC: sulco pós-central; STS: sulco temporal superior; GFI: giro frontal inferior; GFM: giro frontal médio; GPrC: giro pré-central; GPOC: giro pós-central; GSM: giro supramarginal; GA: giro angular; StC: sutura coronária.

O SC também pode ser identificado por sua relação com a sutura coronária. Sua extremidade superior encontra-se a aproximadamente $5,0 \mathrm{~cm}$ posterior à sutura coronária (bregma $)^{4-6}$. Em cortes tomográficos essa distância é de $4,7 \mathrm{~cm}$. O SC aparece como uma linha oblíqua no sentido póstero-anterior e médio-lateral, próxima ao centro do corte tomográfico paralelo à linha órbito-meatal e a, aproximadamente, $4,7 \mathrm{~cm}$ posterior à sutura coronária ${ }^{2}$ (Fig 4). 
3 - Projeção do SC à frente da pars marginalis

(bracket sign)

As duas PM formam uma linha curva que cruza a linha média, configurando um parêntese (bracket) de cada lado da fissura inter-hemisférica. Em espécimes anatômicos, o SC encontra-se $16 \mathrm{~mm}$ anterior à PM em $96 \%$ dos hemisférios esquerdos e em $100 \%$ dos hemisférios direitos 7 (Fig 3).

Identifica-se a PM na TC, como o sulco junto à linha média, no corte de maior diâmetro biparietal do vértice do crânio (bossas parietais). Imediatamente à frente, encontra-se o SC como uma linha oblíqua. Nas imagens horizontais da RM, a PM encontra-se mais posterior do que nos cortes da TC paralelos à linha órbitomeatal (Fig 4). Ela é bem visibilizada em $95 \%$ na TC, e em 91\%, na RM (Figs 3, 4 e 5) ). $^{3}$

Devido à posição bastante constante da PM, Naidich e Brightbill ${ }^{3}$ aconselham o uso do bracket sign como o primeiro passo para identificar os sulcos e giros da convexidade cerebral. Este sinal é identificado em $87 \%$ na TC e na RM, sendo o mais útil para identificação do SC.

\section{4 - SPoC bífido cercando a pars marginalis}

Em espécimes anatômicos, a extremidade superior do SPoC é bífida (em forma de Y) na maioria dos hemisférios (92\% à esquerda e $84 \%$ à direita) (Fig 3). A extremidade bífida encerra a extremidade lateral da PM em $88 \%$ dos hemisférios esquerdos e em $72 \%$ dos hemisférios direitos ${ }^{7}$. A extremidade medial do SPoC apresenta-se bífida e parece conter a PM em 57\% na TC e em $82 \%$ na RM (Fig 4) 3 .

\section{5 - Concavidade anterior do joelho médio do}

GPrC apontando para o SFS.

O SC, em seu trajeto oblíquo para cima e para trás, apresenta três sinuosidades, descritas por $\mathrm{Broca}^{8}$ como joelhos: superior, de concavidade posterior; médio, de concavidade anterior; e inferior, de concavidade posterior. A sinuosidade ou joelho médio apresenta a forma de $C$, sendo que sua concavidade anterior encontra-se ao nível da extremidade posterior do SFS, como se este a apontasse (Fig 1, 2, 3 e 4).

O GPrC é delimitado posteriormente pelo SC e apresenta, portanto, as mesmas sinuosidades ou joelhos deste sulco. $O$ joelho médio do GPrC apresenta nos cortes axiais da TC e da RM configuração em forma de gancho (hook sign) ou C, com a concavidade anterior apontando para o SFS em 96\% dos hemisférios esquerdos e em $100 \%$ dos hemisférios direitos (Fig $4)^{9}$. Corresponde à área motora da mão e localiza-se $38 \mathrm{~mm}$ lateralmente à linha média e $38 \mathrm{~mm}$ posteriormente à sutura coronária ${ }^{10,11}$. A concavidade da face oposta do GPoC corresponde ao córtex da sensibilidade vibratória da mão ${ }^{12}$.

A conformação em gancho (joelho médio) do GPrC, com concavidade apontando para o SFS, é identificada em $83 \%$ dos casos na TC e em 96\% na RM $^{3}$.

\section{6 - GPoC mais estreito que o GPrC}

O GPoC é mais estreito que o GPrC, sendo esta relação útil para identificar estes giros e o $\mathrm{SC}^{2}$ (Figs 1 e 2). A largura da porção superior do GPrC é de 13 $\mathrm{mm}$ e a do GPoC de $11 \mathrm{~mm}^{7}$. O GPoC é mais estreito que o GPrC em $87 \%$ na TC e em $98 \%$ na RM. Esse sinal é eficaz para identificar o SC em $79 \%$ na TC e em $98 \%$ na RM (Figs 4$)^{3}$.

\section{7 - Término da extremidade anterior do sulco \\ intraparietal no sulco pós-central}

O SIP aparece no corte axial de mais largo diâmetro biparietal (bossas parietais) próximo à parede óssea, como linha arciforme, de concavidade lateral. Imaginando o corte axial como o mostrador de relógio, os SIP localizam-se nas duas linhas oblíquas das 9-7 e 35 horas. As PM localizam-se na linha (diâmetro) horizontal 9-3 horas e servem como segunda referência para o SIP, pois coincidem aproximadamente com as extremidades anteriores deste sulco. Nos cortes horizontais da RM, o alinhamento do SIP no mostrador de relógio desloca-se ao longo das duas linhas oblíquas 8-7 e 4-5 horas (Figs 3 e 4) . $^{3}$.

Em espécimes anatômicos, o SIP termina anteriormente no SPoC em $72 \%$ dos hemisférios esquerdos e em $64 \%$ dos direitos ${ }^{7}$. Esse sinal é identificado em $71 \%$ na TC e em $96 \%$ na $\mathrm{RM}^{3}$.

\section{8 - Sinal da deflexão em torno da pars \\ marginalis (pars deflection sign)}

Iwasaki et al. (1991) usaram as digitações da substância branca para definir a anatomia dos giros. Esse método, entretanto, mostrou-se pouco útil ${ }^{3}$. Em uma variação desse método, Naidich e Brightbill ${ }^{3}$ definiram um padrão da substância branca para identificar a PM nos cortes axiais da TC e da RM e propuseram o uso do sinal da deflexão em torno da PM.

O GFS, o GPrC e o GPoC convergem, junto à borda súpero-medial do hemisfério cerebral, para formar o lóbulo paracentral, e defletem-se em torno da PM (Figs 3). Este sinal possibilita a identificação da PM em $67 \%$ na TC e, em 69\%, na RM³ (Fig 4).

\section{CONVEXIDADE CEREBRAL BAIXA (LATERAL OU PERISILVIANA)}

A identificação dos sulcos e giros na convexidade 
cerebral baixa deve ser feita de forma seqüencial no sentido ântero-posterior devido às variações serem menores na parte anterior do hemisfério cerebral. As seguintes referências são usadas para a identificação sistemática dos sulcos e giros perisilvianos nos cortes sagitais laterais da $\mathrm{RM}^{13}$.

$1-S L$

A extremidade anterior do SL apresenta dois ramos (horizontal anterior e ascendente anterior) em forma de $\mathrm{Y}$, que penetram no GFI, delineando a parte triangular deste giro. A extremidade posterior curvase para cima, formando o ramo ascendente posterior, que penetra no lóbulo parietal inferior (Figs 1 e 5).

\section{2 - GFI}

O GFI aparece na extremidade anterior do corte sagital lateral, entre o SL, o SFI e o SPrC, como um $M$, formado pelas partes orbital, triangular e opercular. O ramo horizontal anterior do SL separa a parte orbital da triangular e o ramo ascendente anterior separa esta última da parte opercular. Ou seja, sobre o Y dos ramos anteriores do SL forma-se o M do GFI (Figs 1, 2 e 5).

\section{3 - GFM}

O GFM, delimitado pelos SFS e SFI, funde-se posteriormente com o GPrC, interrompendo o SPrC e dividindo-o em um segmento superior e outro inferior (Figs 1, 2 e 5).

\section{4 - Bifurcação do SFI para formar o SPrC}

O SFI termina posteriormente no ramo inferior do SPrC. Este último situa-se imediatamente posterior ao $\mathrm{Y}$ (formados pelos ramos horizontal anterior e ascendente anterior do SL) ou à parte opercular do GFI (Figs 1 e 5).

\section{5 - Relação do SPrC, do SC e do SL com a sutura coronária}

A extremidade inferior da sutura coronária encontra-se sobre o SL, sendo que os ramos anteriores horizontal e ascendente deste sulco localizamse, respectivamente, à frente e junto desta sutura ${ }^{6}$. A metade inferior do SPrC encontra-se posterior à sutura coronária ${ }^{14,15}$.

$\mathrm{Na} \mathrm{RM}$, a sutura coronária aparece como interrupção da díploe. Nos cortes sagitais laterais (perisilvianos), o SPrC encontra-se posterior à linha imaginária vertical que liga a marca da sutura coronária, na tábua óssea, ao SL. As extremidades inferiores do SC e da sutura coronária distam de $2,8 \mathrm{~cm}^{4}$ (Fig 5).

\section{6 - Giro subcentral}

As extremidades inferiores do GPrC e do GPoC fundem-se sob o SC para formar o giro subcentral, de concavidade superior (Figs 1, 2 e 5).

\section{7 - GPoC mais estreito que o GPrC}

Da mesma forma que nos cortes axiais, esta relação é também útil nos cortes sagitais para identificar o GPrC, o GPoC e o SC (Figs 1, 2 e 5) ${ }^{16}$.

8 - GSM sobre o ramo posterior ascendente do $S L$

O GSM, em forma de ferradura, contorna o ramo posterior ascendente do SL, formando a porção anterior do lóbulo parietal inferior (Figs 1 e 5).

9 - GA sobre a extremidade posterior do STS

O GA, também em forma de ferradura, contorna a extremidade posterior do STS, formando a porção posterior do lóbulo parietal inferior (Figs 1 e 5).

\section{0 - Lobo temporal}

Nos cortes sagitais laterais, encontram-se, paralelos e inferiores ao SL, o GTS, o GTM e o GTI, separados pelos STS e STI. A extremidade posterior do GTS liga-se ao lóbulo parietal inferior. O GTM e o GTI ligam-se, posteriormente, ao lobo occipital (Figs 1 e 5).

A linha imaginária que separa os lobos temporal e occipital pode ser traçada no corte sagital lateral da RM a partir de referências ósseas. O sulco parietooccipital encontra-se de 1 a $5 \mathrm{~mm}$ à frente da sutura lambdóide ${ }^{6,15}$. A incisura pré-occipital encontra-se a aproximadamente $3 \mathrm{~cm}$ posterior ao ponto mais lateral da borda superior da pirâmide petrosa ${ }^{14}$. A linha de divisão liga a marca da sutura lambdóide ao ponto da borda ínfero-lateral do hemisfério cerebral, situado $3 \mathrm{~cm}$ posteriormente ao ponto mais superior e posterior do osso petroso.

\section{DISCUSSÃO}

A PM (bracket sing) e a conformação sigmóide (joelho médio) do GPrC e do SC (hook sign) constituem as referências mais consistentes para localizar os sulcos e giros da convexidade cerebral alta nos cortes axiais da TC e da RM (figura 4). A análise deve começar pela identificação dessas referências ${ }^{3}$.

Na convexidade cerebral baixa ou perisilviana, a localização deve ser feita no corte sagital lateral da $\mathrm{RM}$, no sentido ântero-posterior, identificando, na seguinte seqüência: 1) a fissura silviana; 2) o GFl; 3) o GFM; 4) o SFI e o segmento inferior do SPrC; 5) a totalidade do SPrC; 6) o GPrC; 7) o SC; 8) o giro subcentral; 9) o GPoC; 10) ० SPoC; 11) o GSM; 12) o 
STS; 13) o GA; 14) o SIP; e 15) o lóbulo parietal superior (figuras 5$)^{17}$. O uso conjunto dessas referências possibilita a identificação dos sulcos e giros da convexidade cerebral em $90-95 \%$ dos casos $^{3}$.

A anatomia da convexidade cerebral apresenta grande importância prática, tanto em clínica como em cirurgia. A localização das lesões da convexidade cerebral nos exames de imagem permite fazer a correlação clínico-anatômica (correlação dos sinais e sintomas com a topografia). Essa localização permite também programar o acesso cirúrgico, pois os principais sulcos podem ser representados na superfície craniana através dos métodos clássicos da topografia cranioencefálica ${ }^{18}$.

O conceito de acesso microcirúrgico através das cisternas subaracnóideas e das fissuras, especialmente a fissura silviana, foi estendido mais recentemente aos sulcos (via transsulcal) ${ }^{19}$. Na abordagem transsulcal, a abertura do sulco é seguida de incisão cortical no fundo do sulco e dissecção até o alvo cirúrgico. A utilização da via transsulcal permite minimizar o trauma cirúrgico ao cérebro normal.

Qualquer lesão na base de um sulco pode ser abordada por via transsulcal. Três sulcos são especialmente usados para abordar lesões cerebrais profundas: o SFS, o STS e a junção SIP/SPoC ${ }^{20}$.

O SFS dá acesso ao corno frontal do ventrículo lateral. O STS dá acesso à amigdala, ao hipocampo e ao corno temporal do ventrículo lateral. A secção da substância branca no fundo da junção SIP/SPoC leva ao trígono do ventrículo lateral ${ }^{20}$.

Os sulcos e giros da convexidade cerebral formam um padrão reconhecível na TC e na RM. Nenhum critério morfológico (referências ou sinais) proposto para identificar os sulcos e giros da convexidade cerebral na TC e na RM é preciso de forma isolada. Entretanto, o conjunto dos mesmos forma um sistema simples, integrado e de fácil aplicação, que pos- sibilita a identificação dos sulcos e giros da convexidade cerebral na quase totalidade dos casos.

\section{REFERÊNCIAS}

1. Chi JG, Dooling EC, Gilles FH. Gyral development of the human brain. Ann Neurol 1977;1:86-93 .

2. Kido DK, LeMay M, Levinson AW, Benson WE. Computed tomographic localization of the precentral gyrus. Radiology 1980;135:373-377.

3. Naidich TP, Brightbill TC. Systems for localizing fronto-parietal gyri and sulci on axial CT and MRI. Int J Neuroradiol 1996;2:313-338.

4. Horsley V. On the topographical relations of the cranium and surface of the cerebrum. In: Cunningham DJ. Contribution to the surface anatomy of the cerebral hemispheres. Dublin: Academy House, 1892:306-355.

5. Rowland LP, Mettler FA. Relation between te coronal suture and cerebrum. J Comp Neurol 1948;89:21-40.

6. Broca P. Sur les rapports anatomiques des divers points de la surface du crâne et des diverses parties des hémisphères cérébraux. Bull Soc d'Anth 1861;2:340.

7. Ono M, Kubik S, Abernathey CD. Atlas of the cerebral sulci. Stuttgart: Georg Thieme Verlag, 1990.

8. Broca P. Mémoires sur le cerveau de l'homme et des primates. Paris: Reinwald, 1888.

9. Ebeling U, Steinmetz H, Huang Y, Kahn T. Topography and identification of the inferior precentral sulcus in MR imaging. AJNR 1989;10:937-942.

10. Yousry TA, Schmid UD, Jassoy AG, et al.. Topography of the cortical motor hand area: prospective study with functional MR imaging and direct motor mapping at surgery. Radiology 1995;195:23-29.

11. Boling W, Olivier A, Bittar RG, Reutens D. Localization of hand motor activation in Broca's pli de passage moyen. J Neurosurg 1999;91:903-910.

12. Rumeau C, Gouaze A, Salamon G, Laffont J, Gelbert F, Einseidel H, Jiddane M, Farnarier P, Habib M, Perot S. Identification of cortical sulci and gyri using magnetic resonance imaging: a preliminary study. In Gouaze A, Salamon G (eds). Brain anatomy and magnetic resonance imaging. Heidelberg: Springer-Verlag, 1988:11-31.

13. Naidich TP, Valavanis AG, Kubik S. Anatomic relationships along the low-middle convexity: Part I. Normal specimens and magnetic resonance imaging. Neurosurgery 1995;36:517-532.

14. Seeger W. Atlas of topographical anatomy of the brain and surrounding structures. Wien: Springer-Verlag, 1978.

15. Testut L, Jacob O. Traité d'anatomie topographique. Ed. 5. Paris : Gaston Doin Éditeurs, 1929.

16. Meyer J, Roychowdhursy S, Russel E, Callahan C, Gitelman D, Mesulam MM. Location of the central sulcus via cortical thickness of the precentral and postcentral gyri on MR. ANJR 1996;17:1699-1706.

17. Naidich TP, Valavanis AG, Kubik S, Taber KH, Yasargil MG. Anatomic relationships along the low-middle convexity: Part II. Lesion localization. Int J Neuroradiol 1997;3:393-409.

18. Gusmão S, Silveira RL, Cabral G, Arantes A. Topografia craniencefálica: aplicações neurocirúrgicas. Arq Bras Neurocir 1998;17:59-71.

19. Yasargil MG, Cravens GF, Roht P. Surgical approaches to "inaccessible" brain tumors. Clin. Neurosurg 1988;34:42-110.

20. Harkey HL, Al-Mefty O, Haines DE, Smith RR. The surgical anatomy of the cerebral sulci. Neurosurgery 1989;24:651- 654 . 